\title{
Surface Tension of Magneto-Rheological Fluids
}

\author{
Shai Rahimi and Daniel Weihs* \\ Faculty of Aerospace Engineering, Lady Davis Bldg., Technion, Israel Institute of Technology, Haifa 32000, Israel
}

(Received 21 October 2015, Received in final form 21 May 2016, Accepted 23 May 2016)

\begin{abstract}
Surface tension is a major factor in the thermodynamics as well as fluid properties of Magneto-Rheological Fluids (MRF). We measured the surface tension of an MRF using two different methods. A wettability characterization based on contact angles measurements for the fluid interacting with two different surfaces was conducted. A hydrocarbon based commercial MRF with more than $80 \%$ solid weight, placed on quartz and poly-tetra-fluoroethylene (PTFE) surfaces was used. We measured the fluids' surface tension value by means of contact angles measurements and by the falling drop method.
\end{abstract}

Keywords : rheology, magnetic fluids, surface tension

\section{Introduction}

Magneto-rheological fluids (MRF) are two-phase nonNewtonian colloidal suspensions containing micrometer scale permanently magnetized particles in oil or water surroundings. These fluids can flow in response to forces applied by a magnetic field creating a strong paramagnetic directional response resulting from the particles alignment in the fluid [1].

Surface tension is an important physical property which is directly related to the thermodynamic characteristics of the fluid. Additionally, surface tension measurements yield data which are essential for analyzing free surface flow patterns of any fluid and particularly instabilities, wetting behavior and liquid-air interface processes such as jet breakup, spraying technology and droplet impact onto surfaces. A variety of techniques has been established to measure surface tension, such as using direct measurements of capillary pressure, and employing analysis of equilibrium between capillary and gravity forces .

Only few studies deal with surface tension measurements of MRFs. Dababneh et al. [2] measured the surface tension of a magnetite $\left(\mathrm{Fe}_{3} \mathrm{O}_{4}\right)$ based MRF using the glass capillary tube method. They obtained an empirical relation describing an exponential increase of the surface tension with an increase of the concentration of magnetite particles

(C)The Korean Magnetics Society. All rights reserved.

*Corresponding author: Tel: +972-48-293-806

Fax:+972-48-295-060, e-mail: dweihs@tx.technion.ac.il in the fluid. Amin et al. [3] obtained accurate values of surface tension utilizing the Taylor wavelength obtained from measurements of incipient fluid instability limits. These were found to be in good agreement with a tensiometer measurement. Flament et al. [4] determined surface tension by two methods: First, using a confined twodimensional geometry based upon a surface instability at the interface of a magnetic liquid and organic fluid in a vertical magnetic field and second, finding the deformation of a magnetic droplet on a plane surface under the influence of a horizontal magnetic field.. Racuciu et al. [5] used the stalagmometric method to measure the surface tension of ferrofluids containing super paramagnetic $\mathrm{Fe}_{3} \mathrm{O}_{4}$ nanoparticles stabilized with citric acid.

In this study we present results of MRF surface tension characterization by measuring contact angles. This is based on the the method of Fowkes $[6,7]$ who assumes that surface forces are additive and their geometric mean is used for the adhesion work of each type of force. This method enables to determine the surface tension by an experimentally isolated single parameter for each MRFsurface couple, i.e. measurements of the contact angles regardless of mechanical and gravitational effects.

\section{Theoretical Background}

\subsection{Surface tension evaluation by Fowkes' model}

Defining an additive model, Fowkes [6, 7] divides the surface energies or forces into a dispersive component and a polar component 


$$
\gamma_{1}=\gamma_{1}^{d}+\gamma_{1}^{p}
$$

The combination of additivity (Eq. 1) with the geometric mean for each type of force using the Young-Dupré [6] equation yields

$$
\begin{aligned}
& \gamma_{1}\left(\cos \theta_{A}+1\right)=2 \sqrt{\left.\gamma_{1}^{d} \gamma_{2}^{d}\right|_{A}}+2 \sqrt{\left.\gamma_{1}^{P} \gamma_{2}^{P}\right|_{A}} \\
& \gamma_{1}\left(\cos \theta_{B}+1\right)=2 \sqrt{\left.\gamma_{1}^{d} \gamma_{2}^{d}\right|_{B}}+2 \sqrt{\left.\gamma_{1}^{P} \gamma_{2}^{P}\right|_{B}}
\end{aligned}
$$

or

$$
\begin{aligned}
& \left(\gamma_{1}^{d}+\gamma_{1}^{p}\right)\left(\cos \theta_{A}+1\right)=\Gamma_{A}^{d} \sqrt{\gamma_{1}^{d}}+\Gamma_{A}^{p} \sqrt{\gamma_{1}^{p}} \\
& \left(\gamma_{1}^{d}+\gamma_{1}^{p}\right)\left(\cos \theta_{B}+1\right)=\Gamma_{B}^{d} \sqrt{\gamma_{1}^{d}}+\Gamma_{B}^{p} \sqrt{\gamma_{1}^{p}}
\end{aligned}
$$

Where

$$
\begin{aligned}
& \Gamma_{A}^{d}=2 \sqrt{\left.\gamma_{2}^{d}\right|_{A}}, \Gamma_{A}^{p}=2 \sqrt{\left.\gamma_{2}^{p}\right|_{A}}, \Gamma_{B}^{d}=2 \sqrt{\left.\gamma_{2}^{d}\right|_{B}}, \\
& \Gamma_{B}^{p}=2 \sqrt{\left.\gamma_{2}^{p}\right|_{B}}
\end{aligned}
$$

Solution of Eq. (1) and (4-5) yields components of $\gamma_{1}^{d}$ and $\gamma_{1}^{p}$ as a function of wetting angles obtained from two different fluid -surface combinations as:

$$
\begin{gathered}
\left.\frac{\Gamma_{A}^{p}\left(\Gamma_{A}^{p} \Gamma_{B}^{d}-\Gamma_{A}^{d} \Gamma_{B}^{p}\right) \Psi_{B}+\Gamma_{B}^{p}\left(\Gamma_{A}^{d} \Gamma_{B}^{p}-\Gamma_{A}^{p} \Gamma_{B}^{d}\right) \cdot \Psi_{A}}{\left.+\Gamma_{B}^{p 2}\right) \Psi_{A}{ }^{2}-2\left(\Gamma_{A}^{d} \cdot \Gamma_{B}^{d}+\Gamma_{A}^{p} \cdot \Gamma_{B}^{p}\right) \Psi_{A} \Psi_{B}+\left(\Gamma_{A}^{d 2}+\Gamma_{A}^{p 2}\right) \Psi_{B}^{2}}\right]^{2} \\
\gamma_{1}^{p}=\left[\frac{\Gamma_{A}^{d}\left(\Gamma_{A}^{p} \Gamma_{B}^{d}-\Gamma_{A}^{d} \Gamma_{B}^{p}\right) \Psi_{B}+\Gamma_{B}^{d}\left(\Gamma_{A}^{d} \Gamma_{B}^{p}-\Gamma_{A}^{p} \Gamma_{B}^{d}\right) \Psi_{A}}{\left(\Gamma_{B}^{d 2}+\Gamma_{B}^{p 2}\right) \Psi_{A}{ }^{2}-2\left(\Gamma_{A}^{d} \cdot \Gamma_{B}^{d}+\Gamma_{A}^{p} \cdot \Gamma_{B}^{p}\right) \Psi_{A} \Psi_{B}+\left(\Gamma_{A}^{d 2}+\Gamma_{A}^{p 2}\right) \Psi_{B}^{2}}\right]^{2}
\end{gathered}
$$

Where $\Psi_{\mathrm{A}}=1-\cos \theta_{\mathrm{A}}, \Psi_{\mathrm{B}}=1-\cos \theta_{\mathrm{B}}$

\subsection{Surface tension measurement based on falling drop method}

Here we assume quasi-static equilibrium as the MRF is flowing through the capillary at a slow enough flow rate. As the drop volume $\mathrm{V}$ grows its gravity force is balanced by the vertical component of the surface tension force that holds the drop at the contact line of a circle diameter, $\mathrm{D}_{\mathrm{C}}$, at the tip of the capillary. The surface tension is estimated by (Eq. 10)

$$
\gamma_{1}=\frac{g \rho V}{\pi D_{C}}
$$

As the drop grows, eventually the drop weight, $w=$ $g \rho V$, overcomes the vertical component of the surface tension force, $F_{\sigma}=\pi \gamma_{1} D_{C}$, and the drop detaches and falls off, enabling an independent estimation of the surface tension.

\section{Experimental Setup}

An automated advanced goniometer imaging system (manufactured by Rame-Hart, model No. 500-00-220) was used for measuring the MRF wetting angles on the different surfaces. The experimental layout appears in Fig. 1.

\subsection{Materials}

A commercial hydrocarbon-based MRF-132AD magnetofluid (Lord-Rheometrics) was used. This fluid has density of $3.09 \mathrm{gm} / \mathrm{cc}$ and contains $81.64 \%$ by weight magnetically polarized particle solids of $8.87 \times 10^{-6} \mathrm{~m}$ mean size with a range of $2-20 \times 10^{-6} \mathrm{~m}$. We performed all experiments at controlled temperature of $21^{\circ} \mathrm{C}$. The materials used as adhesion surfaces were Quartz and PTFE, chosen specifically as these are commonly used industrial materials with different dispersion surface energy components and are rigid with a well-defined smoothness geometry. The RMS roughness of the surfaces, as measured by an atomic force microscope, was less than $20 \mathrm{~nm}$ (Fig. 2). The roughness can be correlated to the surface tension by well established methods, see ref. [9] for example.

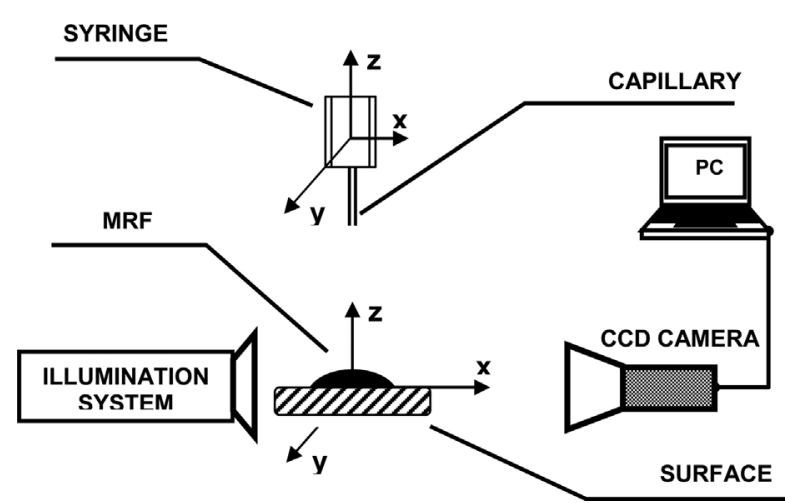

Fig. 1. Schematic diagram of the experimental setup.

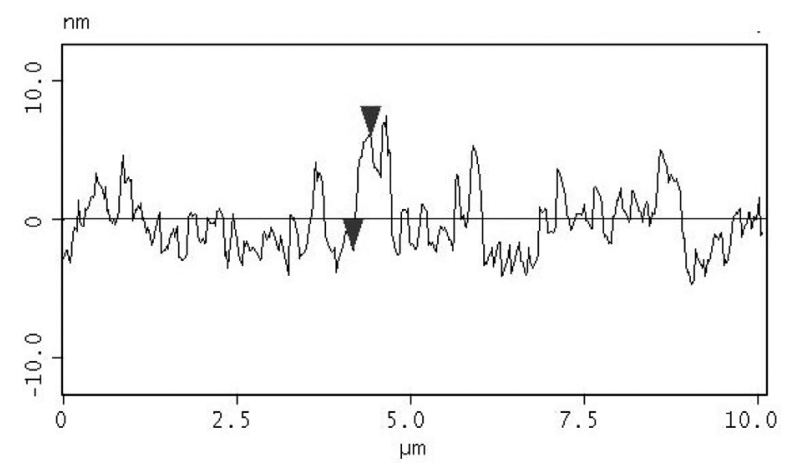

Fig. 2. A typical measurement of Surface roughness of PTFE by AFM. 


\subsection{Wetting angle measurements}

The solid substrates were cleaned using isopropyl alcohol and dry air. Several sessile droplets precisely positioned were dropped onto different solid surfaces using a capillary micrometer-syringe (manufactured by Gilmont, model 100-10-20) held on a x-y-z location mechanism. Side views of the sessile drop were recorded by the goniometer equipped 23X magnification digital CCD camera $(640 \times$ 480 resolution) using backlight illumination. The wetting angles were determined by the image processing code (DROP Image software) using a least squares curve fit numerical approximation to a circular profile of the sessile drop close (only 50 to 100 points) to the baseline at the contact point. The system calibration was conducted using a $4 \mathrm{~mm}$ diameter stainless-steel sphere known object in order to calculate the size of the pixels in the vertical and horizontal directions. Further details may be found in Ref. [10].

\section{Results and Discussion}

Figure 3 presents a three-dimensional description, based on a solution (Eqs. 1, 8, 9) of surface tension for the MRF vs. wetting angles coordinates of the PTFE and quartz surfaces. The dispersion surface energy component, $\gamma_{2}^{d}$, and the polar surface energy component, $\gamma_{2}^{p}$, for the quartz (A) and PTFE (B) substrates used for this analysis are respectively [9]: $\left.\gamma_{2}^{d}\right|_{\mathrm{A}_{p}}=24.79 \mathrm{mN} / \mathrm{m},\left.\gamma_{2}^{p}\right|_{\mathrm{A}}=45 \mathrm{mN} /$ $\mathrm{m},\left.\gamma_{2}^{d}\right|_{\mathrm{B}}=16.86 \mathrm{mN} / \mathrm{m},\left.\gamma_{2}^{p}\right|_{\mathrm{B}}=0.09 \mathrm{mN} / \mathrm{m}$. Wetting angles $\theta_{\mathrm{A}}$ and $\theta_{\mathrm{B}}$ range from $20^{\circ}-90^{\circ}$. As expected, a monotonic increase in the surface tension with wetting angles growth is found.

In order to determine the MRF wetting angles experimentally, we first positioned a $9.41 \mathrm{mg}$ MRF sessile drop on a balanced PTFE surface in ambient air and room temperature conditions. The side view of the MRF sessile drop on PTFE surface is shown in Fig. 3a. Definitions of

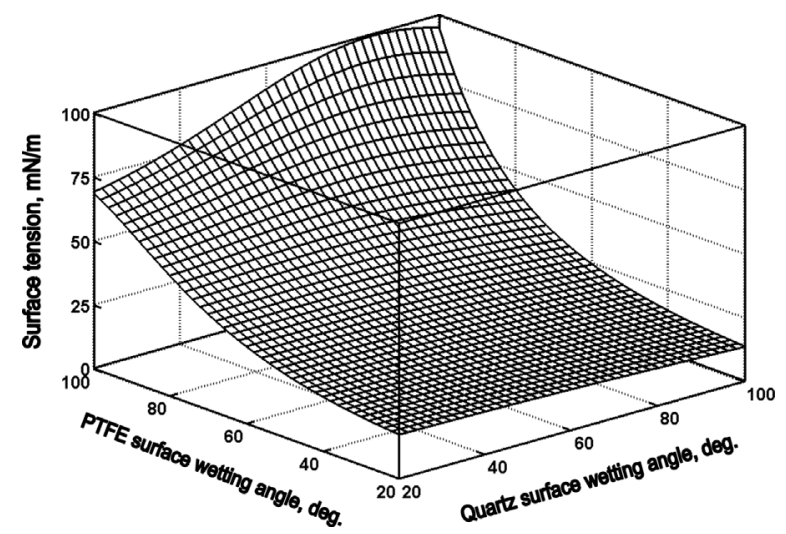

Fig. 3. 3D mapping of the Surface-tension vs. wetting angles.

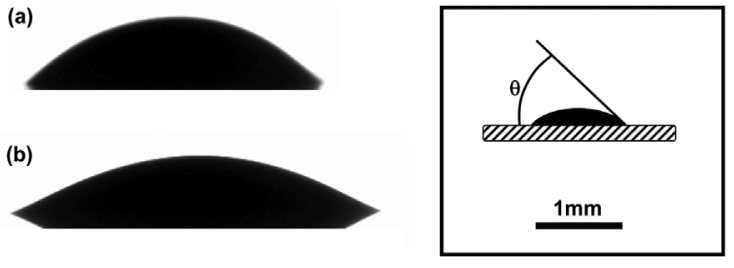

(c)

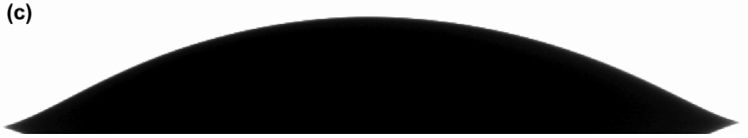

Fig. 4. Side views of MR fluid on different surfaces, ambient air; (a) PTFE surface surface, Fluid mass: $9.41 \mathrm{mg}$, Mean Wetting angle: $49.5^{0}$, sessile drop height: $0.759 \mathrm{~mm}$, sessile drop width: $3.18 \mathrm{~mm}$; (b) Quartz surface, Fluid mass: $8.9 \mathrm{mg}$, Mean Wetting angle: $26.0^{\circ}$, sessile drop height: $0.658 \mathrm{~mm}$, sessile drop width: $4.12 \mathrm{~mm}$; (c) quartz surface, Fluid mass: $65.3 \mathrm{mg}$, Mean Wetting angle: $25.5^{\circ}$, sessile drop height: 1.23 $\mathrm{mm}$, sessile drop width: $8.04 \mathrm{~mm}$.

the wetting angle and a scale bar of $1 \mathrm{~mm}$ are shown in the right top corner of Fig. 4. An average over ten measurements conducted on the same experimental setup on both sides of the $0.759 \mathrm{~mm}$ height and $3.176 \mathrm{~mm}$ diameter sessile drop was conducted, with the standard deviation (STD) based on the entire population given by

$$
\mathrm{STD}=\sqrt{\frac{\sum_{i=1 \rightarrow n}\left(\theta_{i}-\bar{\theta}\right)^{2}}{n}}
$$

Table 1 summarizes the measurement results and their related standard deviation. Wetting angles varied from a minimum value of $49.2^{0}$ to a maximum value of $49.9^{0}$ resulting in mean wetting angle of $49.5^{\circ}$ with standard

Table 1. Contact angles experiments data, MR fluid on PTFE surface, Ambient air, Fluid mass: $9.41 \mathrm{mg}$, Mean Wetting angle: $49.5 \mathrm{deg}$, Height (reduced to 3 significant figures): $0.759 \mathrm{~mm}$, Diameter: $3.18 \mathrm{~mm}$

\begin{tabular}{ccccccc}
\hline \hline No. & Theta $(\mathrm{R})$ & Theta $(\mathrm{L})$ & Mean & STD & Height & Diameter \\
\hline 1 & 49.2 & 49.6 & 49.40 & 0.20 & 0.759 & 3.177 \\
2 & 49.2 & 49.9 & 49.55 & 0.35 & 0.759 & 3.175 \\
3 & 49.2 & 49.7 & 49.45 & 0.25 & 0.759 & 3.177 \\
4 & 49.3 & 49.7 & 49.50 & 0.20 & 0.759 & 3.176 \\
5 & 49.2 & 49.9 & 49.55 & 0.35 & 0.759 & 3.175 \\
6 & 49.3 & 49.8 & 49.55 & 0.25 & 0.759 & 3.176 \\
7 & 49.2 & 49.8 & 49.50 & 0.30 & 0.759 & 3.176 \\
8 & 49.2 & 49.8 & 49.50 & 0.30 & 0.759 & 3.176 \\
9 & 49.3 & 49.8 & 49.55 & 0.25 & 0.759 & 3.175 \\
10 & 49.2 & 49.8 & 49.50 & 0.30 & 0.759 & 3.176 \\
\hline Mean & 49.23 & 49.78 & 49.50 & 0.275 & 0.759 & 3.176 \\
STD & 0.046 & 0.087 & 0.047 & 0.051 & 0.000 & 0.001 \\
\hline
\end{tabular}


Table 2. Contact angles experiments data, MR fluid on quartz surface, Ambient air, Fluid mass: $8.9 \mathrm{mg}$, Mean Wetting angle: $26.0^{\circ}$, Height (reduced to 3 significant figures): $0.658 \mathrm{~mm}$, Diameter: $4.12 \mathrm{~mm}$

\begin{tabular}{ccccccc}
\hline \hline No. & Theta $(\mathrm{R})$ & Theta $(\mathrm{L})$ & Mean & STD & Height & Diameter \\
\hline 1 & 25.8 & 26.1 & 25.95 & 0.15 & 0.658 & 4.123 \\
2 & 25.8 & 26.1 & 25.95 & 0.15 & 0.658 & 4.121 \\
3 & 25.8 & 26.2 & 26.00 & 0.20 & 0.658 & 4.122 \\
4 & 25.8 & 26.1 & 25.95 & 0.15 & 0.658 & 4.122 \\
5 & 25.8 & 26.2 & 26.00 & 0.20 & 0.658 & 4.121 \\
6 & 25.8 & 26.1 & 25.95 & 0.15 & 0.658 & 4.122 \\
7 & 25.8 & 26.1 & 25.95 & 0.15 & 0.658 & 4.122 \\
8 & 25.8 & 26.1 & 25.95 & 0.15 & 0.659 & 4.121 \\
9 & 25.8 & 26.2 & 26.00 & 0.20 & 0.658 & 4.121 \\
10 & 25.8 & 26.2 & 26.00 & 0.20 & 0.659 & 4.121 \\
\hline Mean & 25.8 & 26.14 & 25.97 & 0.17 & 0.658 & 4.122 \\
STD & 0.000 & 0.049 & 0.024 & 0.024 & 0.000 & 0.001 \\
\hline
\end{tabular}

Table 3. Contact angles experiments data, MR fluid on quartz surface, Ambient air, Fluid mass: $65.3 \mathrm{mg}$, Mean Wetting angle: $25.5^{0}$ Height (reduced to 3 significant figures): $1.27 \mathrm{~mm}$, Diameter: $8.04 \mathrm{~mm}$

\begin{tabular}{ccccccc}
\hline \hline No. & Theta $(\mathrm{R})$ & Theta $(\mathrm{L})$ & Mean & STD & Height & Diameter \\
\hline 1 & 25.7 & 25.3 & 25.50 & 0.20 & 1.226 & 8.042 \\
2 & 25.7 & 25.3 & 25.50 & 0.20 & 1.226 & 8.042 \\
3 & 25.7 & 25.3 & 25.50 & 0.20 & 1.225 & 8.041 \\
4 & 25.7 & 25.4 & 25.55 & 0.15 & 1.226 & 8.038 \\
5 & 25.7 & 25.3 & 25.50 & 0.20 & 1.226 & 8.041 \\
6 & 25.7 & 25.3 & 25.50 & 0.20 & 1.226 & 8.042 \\
7 & 25.5 & 25.3 & 25.40 & 0.10 & 1.226 & 8.045 \\
8 & 25.7 & 25.3 & 25.50 & 0.20 & 1.226 & 8.041 \\
9 & 25.7 & 25.3 & 25.50 & 0.20 & 1.226 & 8.040 \\
10 & 25.5 & 25.3 & 25.40 & 0.10 & 1.226 & 8.045 \\
\hline Mean & 25.66 & 25.31 & 25.485 & 0.175 & 1.226 & 8.042 \\
STD & 0.080 & 0.030 & 0.045 & 0.040 & 0.000 & 0.002 \\
\hline
\end{tabular}

deviation less than $0.05^{\circ}$. An average value of $25.75^{\circ}$ with standard deviation of $0.25^{\circ}$ was obtained usingdifferent masses of sessile drops on a quartz surface. The sessile drop masses were $8.9 \mathrm{mg}$ with sessile drop height of $0.658 \mathrm{~mm}$ and diameter of $4.122 \mathrm{~mm}$; and $65.3 \mathrm{mg}$ with sessile drop height of $1.226 \mathrm{~mm}$ and diameter of 8.042 $\mathrm{mm}$. The measurement results and their related standard deviations are summarized in Table 2 and Table 3 respectively. Wetting angles for the $8.9 \mathrm{mg}$ sessile drop varied from a minimum value of $25.8^{0}$ to a maximum value of $26.2^{0}$ with mean of $25.97^{\circ}$ and standard deviation of $0.024^{0}$. Wetting angles for the $65.3 \mathrm{mg}$ sessile drop varied from a minimum value of $25.3^{\circ}$ to a maximum value of $25.7^{0}$ with mean value of $25.48^{0}$ and standard deviation of 0.048 . Side views of the MRF $8.9 \mathrm{mg}$ and $65.3 \mathrm{mg}$ sessile

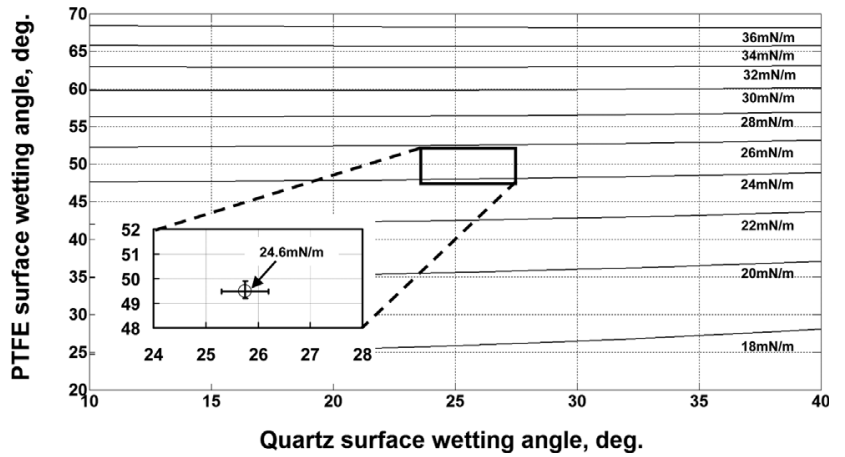

Fig. 5. Surface tension $[\mathrm{mN} / \mathrm{m}]$ iso-lines variation vs. coordinates of quartz and PTFE surface wetting angles.

drops on a quartz surface are shown in Fig. $3 b$ and Fig. 3c respectively.

Figure 5 shows the surface tension iso-lines vs surface wetting angles for both substrates. A wetting angle range of $20^{\circ}$ to $70^{\circ}$ and $10^{\circ}$ to $40^{\circ}$ is presented for PTFE and quartz, respectively. A magnification of the mean wetting angles zone measured for PTFE $\left(49.5^{\circ}\right)$ and for quartz $\left(25.75^{\circ}\right)$ is shown at the right-bottom corner of Fig. 5. This leads to a value of $24.6 \mathrm{mN} / \mathrm{m}$ for the surface tension. Taking into account the extreme combinations of measured values $\left(26.2^{0}, 25.3^{0}\right.$ and $\left.49.2^{0}, 49.9^{0}\right)$ of the wetting angles, in order to examine the deviations from the surface tension value determined from the wetting angles. These gave mean values of $24.475 \mathrm{mN} / \mathrm{m}$ (minimum) and $24.79 \mathrm{mN} / \mathrm{m}$ (maximum). Consequently, the surface tension value estimated by this theory can be determined with less than $1 \%$ error due to wetting angle measurement variations.

Additionally, the MRF surface tension was estimated using the falling drop method MRF falling drop side view sequences (1000 FPS, $0.5 \mathrm{~ms}$ exposure time, $1024 \times 1024$ resolution) from a syringe of $2.1 \mathrm{~mm}$ capillary diameter

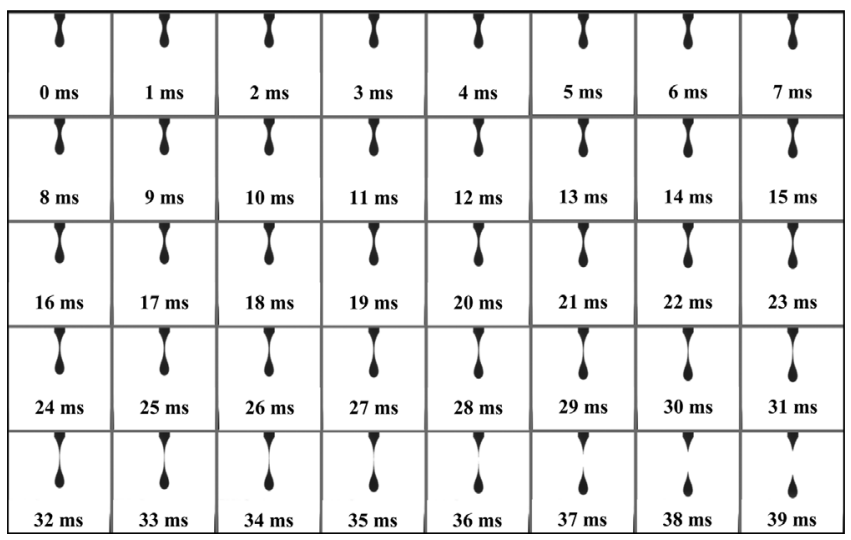

Fig. 6. Falling drop sequences, MR fluid, $D_{c}=2.1 \mathrm{~mm}, 1000$ FPS, $0.5 \mathrm{~ms}$ exposure time, $1024 \times 1024$ resolution. 

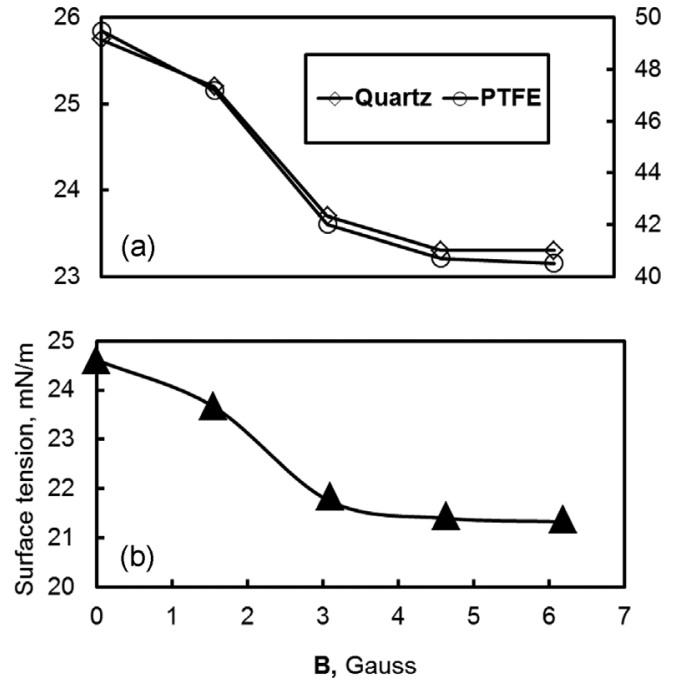

Fig. 7. (a) The contact angles of the MRF 132AD magnetofluid (Lord-Rheometrics) on Quartz (left scale) and PTFE (right scale) versus the magnetic field strength, (b) Surface tension of 132AD magneto-fluid versus magnetic field strength. Triangles are our experimental results and the continuous line is our derived eq. (12).

and droplet mass of $15.8 \mathrm{mg}$, is shown in Fig. 6. From these experimental measurements using Eq. (10) we obtained a surface tension of $23.5 \mathrm{mN} / \mathrm{m}$. A comparison of the results obtained by additive theory $(24.6 \mathrm{mN} / \mathrm{m})$ with those obtained from the falling drop method show very good agreement (less than $5 \%$ difference between methods).

So finally, in Fig. 7, we show the best estimates of wetting angle, for the two surfaces and the resulting measurement of surface tension of the MRF fluid as function of magnetic field strength. The points in Fig. 7b can be connected by a curve (solid line) written as

$$
\sigma(B)=\gamma_{1}^{\infty}+\frac{\gamma_{1}^{0}-\gamma_{1}^{\infty}}{\left(1+0.07 B^{4}\right)}
$$

\section{Conclusions}

We studied the surface tension of MRF using an additive-force model $[6,7]$ and compared with the results of using the falling drop method.

The surface tension value estimated by the additive force model for Magneto Rheological Fluids can be determined with less than $1 \%$ error, mainly resulting from wetting angle measurement variations.

A comparison of the results obtained by the additive theory to these obtained from the falling drop method shows very good agreement (less than $5 \%$ difference).

\section{Nomenclature}

$B \quad$ : Magnetic Induction $(\mathrm{Ga})$

$D_{C} \quad$ : Capillary radius $(\mathrm{m})$

$D_{0} \quad$ : Droplet diameter (m)

$g \quad$ : Gravity constant $\left(\mathrm{m} / \mathrm{s}^{2}\right)$

$h \quad$ : Equilibrium capillary rise $(\mathrm{m})$

$M R(F)$ : Magneto Rheological (Fluid)

$\mathrm{n} \quad$ : Number of measurements

PTFE : Poly Tetra Fluoro Ethylene

STD : Standard deviation (Eq. 10)

$\mathrm{V} \quad$ : Volume $\left(\mathrm{m}^{3}\right)$

$\mathrm{w} \quad$ : Weight $(\mathrm{N})$

$\mathrm{x}, \mathrm{y}, \mathrm{z}$ : Cartesian axes $(\mathrm{m})$

$\gamma_{i} \quad$ : Surface tension by method i

$\Gamma_{j}^{i} \quad$ : Functions defined in Eq. (6)

$\theta \quad$ : Wetting contact angle (deg)

$\bar{\theta} \quad$ : Average wetting contact angle (deg)

$\rho \quad:$ Liquid density $\left(\mathrm{kg} / \mathrm{m}^{3}\right)$

$\rho_{a} \quad:$ Air density $\left(\mathrm{kg} / \mathrm{m}^{3}\right)$

$\Psi_{i} \quad$ : Functions defined in Eq. (8)

\section{Subscripts}

A : : Substance A

B : Substance B

\section{Superscripts}

$d \quad$ : dispersion force

$p \quad$ : polar force

$0, \infty$ : values for $\mathrm{B}=0$ and Asymptotic, respectivelely in eq. (12)

\section{References}

[1] R. E. Rosensweig, Ferrohydrodynamics, Cambridge University Press, Cambridge (1985) pp. 45-61.

[2] M. S. Dababneh, N. Y. Ayoub, I. Odeh, and N. M. Laham, J. Magn. \& Magn Mat. 125, 34 (1993).

[3] M. S. Amin, S. Elborai, S. H. Lee, X. He, and M. Zahn, J. Appl. Phys. 97, 10R308 (2005).

[4] C. Flament, S. Lacis, J. C. Bacri, A. Cebers, S. Neveu, and R. Perzynski, Phys. Rev. E 53, 4801 (1996).

[5] M. Racuciu, D. E. Creanga, and Gh. Calugaru, J. Optoelectronics and Adv. Mat. 7, 2859 (2005).

[6] F. M. Fowkes, J. Phys. Chem. 66, 382 (1962).

[7] F. M. Fowkes, J. Phys. Chem. 67, 2538 (1963).

[8] N. J. Hallab, K. J. Bundy, K. M. O'Connor, R. L. Moses, and J. J. Jacobs, Tissue Eng. 7, 55 (2001).

[9] K. J. Kubiak, M. C. T. Wilson, T. G. Mathia, and P. Carval, Wear 271, 523 (2011).

[10] S. Rahimi, Ph.D Thesis, Technion (2014) 108p. 